\title{
AUTOZENTRIERTE ENTWICKLUNG AUS ETHNO-SOZIOLOGISCHER SICHT DAS BEISPIEL JAPAN
}

\author{
VON INGEBORG Y. WENDT
}

\section{Die Bedeutung autochthoner Strukturen}

Es ist in der Gegenwart kaum noch möglich, die Grundstrukturen und den Grundcharakter einer nicht-europäischen (nicht-nordamerikanischen) Gesellschaft zu erkennen. Großstädte und Touristenzentren in Asien, Afrika, Lateinamerika sind durchsetzt mit europäischnordamerikanischen ${ }^{1}$ Einflüssen. Auf abgelegenen Südsee-Inseln scheint man noch verlorene Paradiese finden zu können, $d$. h. dorthin reisende (und damit relativ wohlhabende) Europäer und Amerikaner können dort Natur und Bewegungsraum in luxuriösem Ausmaß, d. h. Freiheit von den einengenden Regelungen der Großstädte in Industriegesellschaften und von Beton genießen; jedoch auch auf ihnen herrscht selten noch der Urzustand der eingeborenen Bevölkerungen, und diese selbst leben keineswegs in einem ,,Paradies“. Vielmehr bilden sie Inseln nicht nur im Meer, sondern auch im internationalen Gegenwartsgefüge: Von der Weltentwicklung werden sie ausgelassen, und die Möglichkeit der Fortsetzung ihres eigenen alten Weges besitzen sie nicht mehr. Sie bilden eine Art Niemandsland in der Zeit. Dieser Feststellung wird vielleicht entgegengehalten werden, daß sowohl die ,,primitiven“ als auch die ,, alten Hoch"-Kulturen von der europäischen Wissenschaft erfaßt worden sind, erstere in der Ethnologie, letztere in historisch-philologischen Disziplinen wie Indologie und Orientalistik. Beide Kategorien von Wissensvermittlung sind jedoch irrelevant für das Verständnis der Gegenwart: Weder die Kenntnis von Verhaltensweisen einzelner versprengter Stämme von Naturvölkern noch die Kunde von alten Kulturreichen bringt uns nützliche Informationen über die Mentalität, die Verhaltensmodi, die Wertsysteme der die internationale Gegenwart mitgestaltenden nicht-europäischen Völker.

Mit der Gegenwart der Länder befassen sich seit relativ kurzer Zeit die Sozialwissenschaften. Ihnen sind aber durch ihre methodologische Ausrüstung und durch ihre Zielrichtung Grenzen gesetzt. Sie sind weder auf die Erfassung außer-abendländischer Gedankensysteme wie die historisch-philologischen Fächer eingestellt noch auf psychologisches Verständnis der Verhaltensweisen nicht-europäischer Menschen und ihrer autochthonen Gesellschaftsstrukturen wie die Ethnologie, sondern von vornherein auf die Erfassung gegenwärtiger sozio-ökonomischer Strukturen. Diese aber sind seit der Kolonialzeit von europäischen Strukturen überformt; autochthone Elemente scheinen nur noch fragmentarisch durch und bewirken ein um so komplexeres Gesamtbild. Da der Begriffsapparat der Sozialwissenschaften von der europäischen Geistesgeschichte geprägt ist, können sie die komplexen Strukturen nicht-europäischer Gesellschaften nur an europäischen Maßstäben messen und sehen sie deshalb einfach in zeitlichem Verzug.

Den Ergebnissen ethnologischer und historisch-philologischer Forschung nach haben nicht-europäische Kulturen jedoch andersartige Gedanken- und Werttraditionen als das Abendland. In der Anwendung der kulturindividuellen europäischen Maßstäbe liegt dem-

1 Wenn im Folgenden „,europäisch“ oder ,,west-europäisch“ gesagt wird, so ist damit auch ,,nord-amerikanisch“ gemeint, da Nord-Amerika von Europäern geschaffen und politisch, wirtschaftlich und kulturell eine jüngere Version Europas ist. 
nach ein Fehler. In Europa erfolgte die Wirtschaftsentwicklung in gerader Linie aus der geistesgeschichtlichen heraus. Die Naturwissenschaften wuchsen an Bedeutung und Einfluß über Theologie und Philosophie hinaus und spalteten sich von deren Wertvorstellungen ab, und innerhalb der Naturwissenschaften verlagerte sich der Akzent in zunehmendem Maße auf deren vordergründig-technische Zweige. Die ,,industrielle Revolution“ war das folgerichtige Ergebnis dieser Entwicklung. Ganz anders war es in den nicht-europäischen Kulturen. Im Verlauf ihrer kulturellen Entwicklungen spalteten sich philosophische und naturwissenschaftliche Tendenzen nicht voneinander $a b$, und innerhalb der letzteren waren Zweige wie Mathematik und Astronomie von Interesse, nicht ein mechanisches Perpetuum mobile.

Um die autochthonen Strukturen nicht-europäischer Völker und damit die Voraussetzungen wirtschaftlicher Entwicklung erkennen zu können, muß ethnologische und sozialwissenschaftliche Forschung kombiniert werden. Die Realgeschichte nicht-europäischer Völker muß berücksichtigt werden, d. h. die politische und wirtschaftliche Geschichte der jüngeren Jahrhunderte - weniger die Kulturgeschichte früherer Jahrtausende! -, und zwar nicht nur aus der Sicht europäischer Wissenschaftler, sondern vor allem auch aus der Sicht der Gelehrten in nicht-europäischen Gesellschaften selbst.

\section{Thesen über den Stellenwert von Traditionalismen}

Dem Erscheinungsbild nach, das Japan der weltwirtschaftlichen Betrachtung bietet, ist das Land eine moderne ,,Leistungsgesellschaft “ in demselben Sinne wie die west-europäischen Länder und die USA. Es sind jedoch einige japanische Individualismen bekannt geworden, die nicht in dieses Bild passen, so z. B. die meist lebenslange Bindung eines Arbeitnehmers an eine Firma, eine Gepflogenheit, die nicht durch höhere oder geringere Leistung des einzelnen durchbrochen werden kann, sondern nur durch schwere gesamtwirtschaftliche Krisen. Stellengesuche und -angebote in Form von Zeitungsanzeigen sind in Japan kaum notwendig und Arbeitsämter schon gar nicht. Ausbildungsinstitutionen und Firmen stehen untereinander in Kontakt, und außerdem wird auch der einzelne Aspirant durch persönliche Verbindungen vermittelt. Westliche Beobachter meinten lange mit dem Unterton der Anrüchigkeit, in Japan seien „Beziehungen“ alles. Ähnlich wurde auch von den japanischen Geschenken gesprochen, die grundsätzlich in der Nähe von Korruption gesehen wurden. Diese Beurteilung entspringt jedoch einer Projektion europäischer Vorstellungen und Praktiken. Das Netz der japanischen „Beziehungen“, die Gepflogenheit des Schenkens, die Bindung zwischen Firma und Arbeitnehmer basieren auf einem elementar-humanen, lebendigen Gesellschaftskodex. Für die Firmenleitung z. B. ist nicht eine einzelne Eigenschaft wie Fleiß oder Intelligenz maßgebend, sondern vielmehr die Gesamtpersönlichkeit und deren Integrität. Deshalb verläßt sie sich bei Einstellungen auf die Empfehlung von Bekannten oder Verwandten, ähnlich wie es auch prospektive Ehepartner tun.

Der spezifische japanische Gesellschaftskodex spielt sogar bei etwas so Materiell-Konkretem wie der Zahlung von Löhnen und Gehältern eine Rolle. Bei einem internationalen Vergleich mag das Niveau der Vergütungen der japanischen Arbeitnehmer immer noch relativ niedrig liegen; der Vergleich hat jedoch im Ansatz liegende Schwächen. Er erfaßt nicht das spezifisch japanische ,System“ von Zuwendungen und Geschenken bei persönlichen Aalässen, das nirgends offiziell festgelegt und deshalb statistischer Berechnung nicht zugänglich ist. Dieser Hintergrund muß auch bei der Beurteilung der Kranken- und Altersversorgung berücksichtigt werden. 
Ein Beispiel für den sich von der europäischen Art unterscheidenden Umgang mit Geld überhaupt in Japan ist es, daß in der Regel einer die Gesamtrechnung begleicht, wenn eine Gruppe von Freunden essen geht. Getrennte Rechnung ist die Ausnahme und wird ,dutch account" genannt, weil die Japaner diese Sitte bei den vom 17. - 19. Jh. im Land geduldeten Holländern beobachtet haben. Großzügigkeit ist in Japan verbreitet. Diejenigen Japaner, die Wechselgeld nicht nachprüfen, sind sicher in der Mehrzahl. Die verschwenderischen Tokyoiten haben im 18. Jh. begonnen, die sparsameren Leute von Osaka zu überflügeln und sind heute im Wirtschaftsleben führend.

Wenn schon der Umgang mit materiellen Gütern in der modernen japanischen Wirtschaftsstruktur individuell und flexibel geblieben ist, dann sind es erst recht die allgemeinen Lebensgewohnheiten. Japaner nehmen z. B. keinen 3/4wöchigen Jahresurlaub, sondern machen häufiger im Jahr mehrtägige Ferien zwecks Ski-Lauf, Baden in heißen Quellen, Besuch von Tempeln oder anderen Kulturstätten - die Eisenbahnen zumindest in Mittel-Japan sind immer voll besetzt - und zu Familienzusammenkünften anläßlich der traditionellen mehrtägigen Jahres-Hauptfeste o-shogatsu (Neujahr) und o-Bon ${ }^{2}$ im Sommer. Ferner gibt es in jedem Monat mindestens einen volkstümlich-religiös begründeten Feiertag, und außerdem nimmt man sich hin und wieder einen Tag frei - wenn gerade nicht viel zu tun ist oder wenn in den letzten Tagen besonders viel zu tun war oder wenn man sich leicht unpäßlich fühlt. Das Ausmaß, in dem in der komplexen modernen Industriegesellschaft Japans die persönliche Note im Leben der Menschen erhalten bleibt, ist für einen Europäer oder jedenfalls für einen Deutschen kaum vorstellbar. Es gibt nie nur eine mögliche Regelung, nicht entweder/oder, sondern immer sowohl/als auch. Man paßt sich im internationalen Verkehr der abendländischen Zeitrechnung an, benutzt aber im inner-japanischen Postverkehr den traditionellen japanischen Kalender (5. 12.79 = 54-12-5). Man kann die chinesisch-japanischen Schriftzeichen von oben nach unten, von rechts nach links, von links nach rechts und den Namen Japans mit chinesischen Schriftzeichen, japanischen Silbenzeichen - unterschiedlich! -, mit lateinischen Buchstaben schreiben und auch unterschiedlich aussprechen (Nippon oder Nihon). Wie die abwechslungsreiche Landschaft und das turbulente Klima mit Taifun und Erdbeben ist auch die Psyche der Bewohner der japanischen Inseln sehr beweglich; die Japaner sind äußerlich viel unterwegs und würden sich innerlich reglementiert fühlen, wenn sie z. B. gezwungen wären, Personalausweise zu führen (Pässe erhalten sie für Auslandsreisen).

Vom Standpunkt einer westlichen ,Leistungsgesellschaft“ aus ist Japan noch immer ,, undurchsichtig"; verständlich wird es erst, wenn man es auf dem Hintergrund seiner autochthonen Gesellschaftsst '"lktur sieht:

In Japan hat die Großfamilie bis heute nicht aufgehört zu bestehen. Zwar hat die industrielle Ballung in der sich von Tokyo über Yokohama, Nagoya, Kyoto, Osaka bis Kobe erstrekkenden Megalopolis auf rund 600 km Küstenstreifen am Pazifik bewirkt, daß eine zusammenlebende Großfamilie in Japan selten geworden ist. Am inneren Zusammenhalt mit allen Konsequenzen hinsichtlich Verantwortung für das Wohlergehen der Familienmitglieder, Loyalität dem Familienältesten gegenüber und auch der Bindung an die Gräber der Vorfahren hat sich bis heute nichts geändert.

Im modernen Leben erstreckt sich eine quasi-familiäre Bindung auf die entstandenen beruflichen Gruppen in Industrie und Universität und auf die Mitglieder einer Gruppe von Freunden, ähnlich wie es in den Krieger- und Feudal-Klans früherer Jahrhunderte gewesen ist.

2 O-Bun ist ein volkstümlich-religiöses Fest, anläßlich dessen die Ahnen ihre noch auf der Erde lebenden Nachkommen besuchen. oshôgatsu und o-Bon sind im modernen japanischen Wirtschaftsleben manifestiert durch Zahlung der 13. und 14. Monatsgehälter und durch steilen Anstieg des innerjapanischen Flugverkehrs. 
Der gesellschaftliche Kodex, auf dem alle diese Eigenschaften direkt oder indirekt basieren, ist ein gegenseitiger Verlaß aufeinander, eine gegenseitige Abhängigkeit voneinander, eine gegenseitige Anlehnung aneinander, ein ,System“, das die gesamte Gesellschaftsstruktur durchzieht, dem der Fabrikdirektor wie der Arbeiter, der Lehrer wie der Schüler, der Ältere wie der Jüngere sich verpflichtet fühlt. Man empfängt und erteilt Gefälligkeiten, man lädt eine Last auf eines andern Schulter und erhält selbst Lasten aufgeladen, man ist ständig, gebend oder empfangend, in nahe menschliche Beziehungen verwickelt, und die jeweilige Funktion hat einen Namen. Wenn man ,,danke" sagt, so heißt das ,,es ist nicht zu Ende" (sumimasen), und der Kern dieses Gesellschaftskodex' ist das oben beschriebene amae (ama-e $)^{3}$, das der japanische Psychologe Doi in mehreren Veröffentlichungen dargestellt und analysiert hat ${ }^{4}$.

Die japanische Wirtschaftsentwicklung widerlegt durch sich selbst die westlich-sozialwissenschaftliche Theorie, nach der die traditionelle Großfamilie entwicklungshemmend wirken würde. Die Stärkung durch den Zusammenhalt und damit gegenseitige Hilfestellung scheint die Belastung durch das Mittragen schwacher Glieder mehr als auszugleichen; und selbst wenn die durch Industrieballung verursachte äußere Auflösung der Großfamilie sich auch auf den inneren Zusammenhalt auswirken würde, so wäre das erst eine Folge der Industrialisierung, sie war nicht ihre Voraussetzung.

Durch historische Entwicklungen bereits widerlegt ist auch die BevölkerungsexplosionsThese. Der These nach muß die Wirtschaftsentwicklung in den Entwicklungsländern stagnieren, weil dort dank der empfangenen Vorteile der westlichen Medizin die Säuglingssterblichkeit sinkt und die Lebenserwartung steigt, während die Bevölkerungen technischindustriell den modernen Stand noch nicht erreicht haben. So einfach kann es jedoch den geschichtlichen Erfahrungen nach nicht sein.

In Japan stieg die Zahl der Bevölkerung von der Mitte des 19. Jahrhunderts bis 1929, also während des Starts und der ersten, grundlegenden Industrialisierungsphase, um mehrals 100 $\%$, nämlich von 30 auf gut 60 Millionen. ${ }^{5}$ Entsprechende Daten bilden in Europa noch eine Alltagserfahrung der älteren Generationen, deren Väter und Mütter oft noch 10 Geschwister hatten.

Außerdem ist der Versuch, Wirtschafts- und Bevölkerungswachstum zu korrelieren, älter als die Fortschritte der Medizin. Schon während der Kolonialepoche wollten britische Kolonialbeamte die wachsende Verelendung der indischen Bevölkerung mit zu hohen Geburtenraten erklären. Romesh Dutt wies damals nach, daß die Bevölkerungswachstumsrate in England höher war als in Indien. ${ }^{6}$

Bevölkerungs- und Wirtschaftswachstum sind zwei Komponenten in einem Gesamtprozeß, sie dürfen nicht isoliert von diesem betrachtet werden. Es ist sehr fraglich, ob der Bevölkerungsanstieg in Europa und in Japan rund 50 Jahre um die Jahrhundertwende etwas mit dem Fortschritt der medizinischen Wissenschaft zu tun hatte. In Japan z. B. besteht ein Zusammenhang zwischen dem ansteigenden Bevölkerungswachstum und der Aufgabe der vorher in einigen Schichten der Bevölkerung praktizierten Sitte der gezielten Familienbegrenzung. ${ }^{7}$ Und umgekehrt hat in Europa der Rückgang des Bevölkerungswachstums nicht erst mit der Entdeckung sicherer Kontrazeptionsmittel eingesetzt.

3 A-ma-e-ru (Verb) heißt: sich verlassen auf, sich anlehnen an, abhängig sein von.

4 Z. B. Doi, Takeo: Anatomy of Dependence. Tokyo 1973.

$5 \mathrm{Vgl}$. Wendt, Ingeborg Y.: Japanische Dynamik und indische Stagnation? Eine Antwort auf theoretische Entwicklungsmodelle. Darmstadt 1978, S. 142.

6 Dutt, Romesh: The Economic History of India. Vol. I: Under early British Rule 1757-1837; Vol. II: In the Victorian Age 1837-1900, 2. Aufl. London 1906. (Indische Neuauflagen durch das Ministry of Information and Broadcasting des Government of India, London 1959, 1963, 1970.)

7 Mabiki = Ausdünnen junger Reispflanzen, übertragen auf den menschlichen Nachwuchs. 
D. L. Umali kommentiert als Stellvertretender Generaldirektor und Regionalpräsident für Asien und den Fernen Osten der Welternährungs- und Landwirtschaftsorganisation (FAO): „,Fruchtbarkeitsraten sind . . . eines der unerschütterlichsten Dinge in der Welt. Trotz der Verfügbarkeit von kontrazeptiven Mitteln bleiben sie hoch, bis der Lebensstandard und das Einkommen sich merklich verbessern. Dann, und nur dann, beginnen sie zu sinken. "8 Vielleicht gehen die natürlichen, sozialen und internationalen Zusammenhänge, ,,Gesetzmäßigkeiten", sogar noch über diese Erklärung hinaus. Die sich industrialisierenden Länder absorbierten damals den Bevölkerungszuwachs durchaus, sozio-ökonomisch betrachtet brauchte die sich formende Struktur ihn, d. h. billige Arbeitskräfte, sogar. Erst recht würde Indien heute Bevölkerungszuwachs brauchen, wenn es dem Land möglich wäre, seine natürlichen Bedingungen wiederherzustellen, seine brachliegende Landwirtschaft wieder aufzubauen.

Wer in niedrigen Höhen über den Subkontinent fliegt, ist überrascht über das Ausmaß der unbebauten, aber bebaubaren Flächen indischen Bodens. Nur weil in der Gegenwart nicht auf Land und Landwirtschaft, sondern ausschließlich auf die überfüllten Großstädte und auf technische Industrialisierung in den Entwicklungsländern geachtet und weil vergessen wird, daß die Industrialisierung in Europa und Japan bei zwar verarmter, aber funktionierender Landwirtschaft erfolgte ${ }^{9}$, kann heute der Bevölkerungszuwachs in den Entwicklungsländern als entwicklungshemmend betrachtet werden.

Uberhaupt herrschen in den Industrienationen falsche Vorstellungen über die Relation von Bevölkerungszahlen und geographischer Ausdehnung in der nicht-europäischen Welt. Kaum ein Europäer ist sich darüber im klaren, daß Japan von Indien so weit entfernt ist wie Indien von West-Europa. Und der internationale Tourist, der nicht darauf käme, die Bevölkerungsdichte von Paris auf ganz Frankreich zu übertragen, hält doch den ganzen indischen Subkontinent für übervölkert, wenn er Bombay und Calcutta sieht. Drittens kommen Verzerrungen der tatsächlichen Gegebenheiten manchmal sogar durch wissenschaftliche Techniken zustande; so ist im Heft 2, 1978, der Zs. ,,Vereinte Nationen“ eine Darstellung der Erde, die den Äquator nicht real-geographisch, sondern wirtschaftspolitisch verlaufen läßt. Zur südlichen Halbkugel gehören dann Länder wie die Türkei, China und Indien, die faktisch nördlich vom Äquator liegen. Da die Bevölkerung dieser südlichen Erd, ,hälfte" mit 2/3 der Weltbevölkerung angegeben wird, ergibt sich daraus eine totale Irreführung hinsichtlich des tatsächlichen Verhältnisses von Bevölkerungszahl zu geographischer Ausdehnung im Norden und im Süden.

Ähnlich unrealistisch, und zwar in doppelter Hinsicht, sind die europäischen Vorstellungen in bezug auf die Bedeutung der Schriftkundigkeit. Zum einen ist Denkvermögen nicht ans geschriebene Wort gebunden, und zum andern müssen nicht alle Ausführenden technischer Arbeiten denken können oder schriftkundig sein. Es ist eine wenig bekannte Tatsache, daß die industrielle Revolution in Europa bei verbreitetem Analphabetismus erfolgte. Noch 1837 ging in England in den Großstädten nur eins von vier oder fünf Kindern zur Schule, vom Land ist gar nicht die Rede. Und noch 1877 schneidet bei einem wissenschaftlichen Vergleich des allgemeinen Bildungsstandes in Japan und in Frankreich das europäische Land schlechter ab $\mathbf{1 0}^{\mathbf{1 0}}$.

$\mathrm{Daß}$ die industrielle Technik heute höher differenziert ist, bedeutet nicht, daß alle einzelnen Bürger der Industrienationen wissenschaftlich-technisch höher differenziert ausgebildet

8 Vgl. die im „Spiegel der Presse“ des BMZ vom 2. 12. 75 wiedergegebene Analyse der Ubervölkerungs-These.

9 Im Jahr 1877 machten in Japan die Steuereinkünfte aus der Landwirtschaft $82 \%$ der gesamten Steuereinnahmen aus, vgl. Wendt, Ingeborg Y.: Die ,, unheimlichen“ Japaner. Stuttgart 1970. S. 94/95. - Ausführlich zu diesem Thema das klassische Werk von Norman, E. Herbert: Japan's Emergence as a Modern State, New York 1940.

$10 \mathrm{Vgl}$. Dore, Ronald: Education in Tokugawa-Japan. Los Angeles 1965. 
sein müßten oder sind. Die medizinische Laborantin z. B. hat nur ein bestimmtes Repertoire von in der Psychologie ,,Wenn-Dann“-Relationen genannten Vorgängen, nicht in Kausalzusammenhängen denken gelernt. Ebenso geht es der Finanzangestellten, die früher einige selbständige Gedankenschritte vollziehen können mußte; sie braucht heute nur die Funktion einiger Knöpfe und Hebel zu kennen, kaum anders als eine Arbeiterin am Fließband, und das könnte sie auch, wenn sie nicht das lateinische Alphabet beherrschte, sondern nur einige geometrische oder Bild-Zeichen voneinander unterscheiden könnte.

Der geschichtlichen Erfahrung nach behindern weder Religion noch Kastensystem noch andere Traditionalismen wirtschaftliche Entwicklung. Z. B. wäre es bis vor nicht langer Zeit in Europa ein Eklat gewesen, wenn ein Berufsoffizier eine Verkäuferin oder die Tochter eines Arztes einen Maurer geheiratet hätte, und noch in der Gegenwart wäre ein als solcher erkenntlicher Arbeiter der Hand in einem der Luxushotels europäischer Großstädte so wenig willkommen wie ein indischer Unberührbarer in bestimmten Tempeln.

\section{Ein entwicklungsgeschichtlicher Vergleich}

Das Kastensystem ist, neben heiligen Kühen, geradezu sprichwörtlich für Stagnation durch Rückschrittlichkeit geworden. Dem steht theoretisch Japan, neben Europa, mit einem rational denkenden Mittelstand gegenüber. Es seien deshalb einige der Grundlinien der Entwicklungsverläufe Japans und Indiens einander gegenübergestellt.

Die Epoche der Abschließung Japans vom 17. bis 19. Jh. war weder politisch noch kulturell noch wirtschaftlich eine solche ,,konfuzianischer Erstarrung“, wie lange angenommen wurde. Die offiziellen vier Stände waren faktisch nicht die einzigen ${ }^{11}$, und außerdem gab es nicht offiziell, aber im praktischen Alltag - soziale Mobilität durch Heirat, Adoption, finanziell begründeten Úbertritt. Die politische Macht war zwar zentralisiert, aber unterschwellig liefen die alten Machtkämpfe weiter, bereitete sich der Machtwechsel vor, der sich am Ende des 19. Jahrhunderts vollzog. Innerhalb des Konfuzianismus gab es unterschiedliche Auffassungen, und ihre Vertreter führten über die 2 1/2 Jahrhunderte des Friedens einen geistigen Kampf, der die sogen. Meiji-Restauration von 1868 mitvorbereitete. Die Finanzkraft verschob sich infolge menschlicher Schwächen: die Herrschenden, d. h. die Angehörigen des Standes der Krieger, gerieten in die Schuld des offiziell untersten Standes der Kaufleute. Die Land- und Stadtbevölkerungen waren auf unterschiedliche Weise in Bewegung: Mehr als tausend Bauernaufstände fanden statt; dagegen lebten die Bürger von Edo (heute Tokyo) in ungeplantem Wohlstand luxuriös und verschwenderisch, gewannen dabei noch an Einfluß und bildeten den Boden für die Entstehung einer volkstümlichen Kunst, zu der z. B. die auch in Europa bekannt gewordenen Holzschnitte mit ihrer sozialen Thematik, vor allem ihren heiteren Aspekten, gehören.

Japan war bis zur Mitte des 19. Jahrhunderts das einzige Land, das weder direkt noch mittelbar von der europäischen Kolonialherrschaft betroffen wurde, und als es 1853/54 unausweichlich mit ihr, in Form der Forderung des amerikanischen Commodore Perry ${ }^{\mathbf{1 2}}$, konfrontiert wurde, war es eine dynamische, intakte Gesellschaft, die bis dahin nach ihren eigenen Antrieben und Bedürfnissen funktioniert hatte und deren materielle Substanz in ihren eigenen Händen geblieben war.

11 Der Rangordnung nach Krieger, Bauern, Handwerker, Kaufleute; daneben die Klöster, die Aristokratie, die hinin oder burakumin (eine Art Unberührbare) u. a.

12 Vgl. Wendt, Die ,unheimlichen“ Japaner S. 70/71. 
Ganz anders Indien. Als Japan in seine gegen die Welt erzwungene Abschließung ging, begannen die Kriege der verschiedenen europäischen Mächte untereinander sowie gegen einheimische Fürsten in Indien sich auszudehnen und zu verschärfen. Auch nach der Etablierung der englischen Ost-Indien-Ges. als territorialer Macht mit der Schlacht von Plassy 1757 gingen die Kämpfe weiter. Neben den Kriegsverwüstungen begannen die wirtschaftlichen und verwaltungstechnischen Maßnahmen der Kolonialmacht ihren Einfluß zu nehmen. Die Tarife des überseeischen wie des innerindischen Handels begünstigten ausnahmslos und in extremem Ausmaß die Ost-Indien-Ges., später die britische Regierung, als Institution und auch deren einzelne Angehörige als Privatpersonen. Der indische Reichtum floß als ,Economic Drain" aus dem Land ab. Ebenso waren die Investitionen auf die Förderung englischer Interessen, etwa den Bau von Eisenbahnen als Verbindungsmittel zwischen englischen Handelszentren, ausgerichtet und nicht auf die Instandhaltung lebensnotwendiger indischer Einrichtungen wie der verschiedenen traditionellen Bewässerungsanlagen.

Die Pacht- und Steuersätze für die indischen Bauern erreichten Höhen, die weder von früheren mohammedanischen Herrschern noch in Europa oder Japan je gefordert worden waren $^{9}$. Die indischen Bauern waren schließlich gezwungen, ihr Vieh und schon die neue Saat zu verpfänden. Da sie keine Reserven mehr besaßen, die einen innerindischen Austausch ermöglichten, und da auch die verschiedenen traditionellen Bewässerungssysteme allmählich verfielen, entstanden bei regionalen Dürren Hungersnöte, denen Millionen von Menschen zum Opfer fielen.

So katastrophal wie die materiellen waren auch die geistigen und die sozialstrukturellen Folgen der Kolonialherrschaft. Indische Bildung, Wertmaßstäbe und soziale Bindungen galten nicht mehr. Schriftkundige und -unkundige waren in derselben Situation: Sie wurden zu bestimmten Handlungen und Arbeitsleistungen gezwungen. Uber viele Jahrhunderte, vielleicht Jahrtausende, hatten die Dorfgemeinschaften den Wechsel aller höheren Regierungseinheiten überdauert und den tragenden Kulturboden Indiens gebildet. Sie waren hochdifferenzierte und gleichzeitig natürliche Selbstverwaltungseinheiten, in denen jedes Mitglied seine Funktion hatte. Unter der britischen Regierung gewann jedoch der rangniederste Kolonialbeamte mehr Autorität als das geachtetste Mitglied der einheimischen Struktur. Die alte indische Dorfgemeinschaft, der Kern der indischen Sozialstruktur, wurde zerstört. Das vorkoloniale Indien hatte nicht nur seine angemessenen Bewässerungssysteme und eine tragende Agrarstruktur besessen; es hatte mit seinen Roh- und Fertigwaren auch erfolgreich am alten orientalischen Markt teilgehabt. Unter der englischen Kolonialherrschaft wurde dieser Handel ausgelöscht. ,,Ein neuer Handel begann in Indien zu blühen: Handel im Rahmen einer dem Land übergelagerten künstlichen Fremdstruktur, die die indischen Menschen ausschaltete, sich aber der indischen Materialien bediente ${ }^{\mathbf{1 3}}$."

Wenn die Anfänge technisch-industrieller Entwicklung in Indien und in Japan verglichen werden sollen, dann muß die japanische Situation nach 1853/54 - Indien stand zu der Zeit noch fest unter Kolonialherrschaft, der räumlich und zeitlich ausgedehnteste Aufstand gegen die Fremdherrschaft, the Great Mutiny, scheiterte 1857 - der indischen Situation nach dem Zweiten Wieltkrieg, genauer nach Erlangung der Unabhängigkeit gegenübergestellt werden.

Japan konnte den entscheidenden Schritt als eine intakte, funktionsfähige, in seiner Tradition ruhende Einheit tun; es begann seiner eigenen kulturellen Substanz die Ergebnisse europäischer Naturwissenschaft, seiner traditionellen Sozialstruktur die Details europäischer Technik hinzuzusetzen, und diese Entwicklung erfolgte sogar bewußt, nämlich nach dem

13 Wendt, ,Japanische Dynamik ... " S. 123. 
Motto ,,westliche Technik, östliche Moral“. Daneben besaß Japan eine jahrhundertealte materielle Substanz. - Indien dagegen trat sowohl mit einem wirtschaftlichen als auch mit einem sozio-psychologischen Defizit ins internationale Leben ein. Indien war keine ,,intakte, funktionsfähige, in seiner Tradition ruhende" Einheit; seine Sozialstrukturen waren zerstört, seine Wertmaßstäbe verunsichert, sein Kapital abgezogen.

Auf diesem Hintergrund müssen in der Gegenwart die vielzitierten ,,Traditionalismen “ verstanden werden. Die heutige Situation Indiens stellt nicht den Urzustand des Landes dar, sondern ist das Ergebnis jahrhundertelanger Fremdherrschaft. Die Annahme, daß das Kastensystem diese Jahrhunderte überlebt hat, während die Gesamtstruktur zerschlagen wurde, ist unrealistisch. Vermeintliche Anzeichen rigiden Verhaltens auf der Grundlage eines Kastensystems sind vermutlich Äußerungen passiven Widerstandes gegen aufgezwungene und nicht den eigenen Bedürfnissen entsprechende Forderungen.

\section{Die Alternative}

Man hat versucht, die erfolgreiche Wirtschaftsentwicklung Meiji-Japans mit dem Vorhandensein eines Mittelstandes zu erklären. Dem vom Geist innerweltlicher Askese beseelten mittelständischen Unternehmer Max Webers schien in Japan der mittlere Samurai zu entsprechen, der materiell anspruchslos und in geistiger Disziplin durch die Zen-Meditation geschult war ${ }^{\mathbf{1 4}}$. Diese Parallelsetzung ist jedoch weder geistes- noch wirtschaftsentwicklungsgeschichtlich haltbar.

Die Zen-Disziplin war eine auf den inneren Kern des Seins gerichtete konzentrative Anstrengung, bei der Geist und Sinne gesammelt und beherrscht wurden. Die Konzentration konnte zur geistigen Erleuchtung, Satori, führen, in welchem Zustand das Ich Eins mit dem Sein war und die Welt von ihren rationalen Grenzen befreit erlebt wurde, oder zur EinsWerdung des Suchenden mit dem Ziel, so daß ein Bogenschütze mit dem Pfeil das Ziel bei geschlossenen Augen treffen konnte ${ }^{15}$. Diese geistige Disziplin, die sich in vielen Sport- und Kunstformen manifestieren kann, wurde, gemessen an den buddhistischen Lehren zweckentfremdet, von den Samurai im kriegerischen Mittelalter zur Stählung kämpferischer Fähigkeiten und zur Uberwindung der Todesfurcht genutzt. Ókonomische Aspekte wie Sparsamkeit oder Verschwendung hatten in dieser Lebenssphäre keinen Raum.

Wirtschaftsgeschichtlich durchliefen die Samurai vom kriegerischen Mittelalter bis zum Anbruch der Moderne mehrere Transformationen Die Kämpfe unter den Kriegerhäusern endeten mit der Zentralisierung der Macht in den Händen der Tokugawa-Regierung in Edo am Beginn des 17. Jahrhunderts. Der Stand der ,,Krieger“ war nun nur noch nominell ein solcher, denn im abgeschlossenen Japan herrschte Frieden, und strukturierte sich neu. Die oberen Schichten bildeten die Regierung, ein Teil der unteren Schichten wurden deren Beamte, ein anderer Teil verlor seine Stellung und wurde herren- und arbeitslos (ronin), ein weiterer Teil gliederte sich in bürgerliche oder künstlerische Berufe ein.

Bei anhaltendem Frieden und wachsendem Wohlstand wurden Kriegshandwerk und -disziplin zwar nicht in allen Kreisen vergessen, aber sie verloren insgesamt an Bedeutung, und am Ende der Tokugawa-Epoche formte sich der Stand der ,,Krieger" noch einmal neu. Die vierbis fünfhunderttausend mittleren Samurai, die nun in moderne Berufe gingen, müssen die von McClelland gemeinten ,,Puritaner" gewesen sein, aber sie waren, nach 21/2 Jahrhunder-

14 Vgl. McClelland, David: Die Leistungsgesellschaft, Stuttgart 1966.

15 Vgl. Herrigel, Eugen: Zen in der Kunst des Bogenschießens 3. Aufl. München-Planegg 1953. 
ten Frieden und Wohlstand, weder mehr die Zen-geschulten Samurai des Mittelalters, noch wurden sie ,„Unternehmer“.

Die Unternehmer-Funktion spaltete sich am Beginn der Industrialisierung in Meiji-Japan in mindestens vier Teilfunktionen auf: die Hauptinitiative hatte die planende Regierung; an zweitwichtigster Stelle standen Großkapitalisten verschiedener Herkunft, die Banken und Finanzierungsinstitute formten; als nächstes kamen die Unternehmer-Familien, die noch in direkter Linie aus dem kriegerischen Mittelalter herüberreichten, und schließlich spielten auch die mittleren Samurai eine Rolle: Sie wurden leitende Angestellte in den von der Regierung initiierten und von den Finanziers betriebenen Großunternehmen. Zur Kapitalakkumulation hätte diese Gruppe der an der Industrialisierung Beteiligten keine Möglichkeit gehabt. Die mittleren Samurai erhielten eine zu geringe Abfindung für die Aufgabe ihrer bisherigen Existenzform, als daß sie damit ein Unternehmen hätten gründen können, und außerdem waren sie es nicht gewöhnt, rational mit Geld umzugehen. Die wenigen mittleren Samurai, die trotzdem Unternehmer oder Kaufleute wurden, scheiterten so oft, daß das Geschäft eines Kriegers, bushi no shôhô, sprichwörtlich für von vornherein zum Mißerfolg verurteilte, dilettantische Unternehmen wurde ${ }^{\mathbf{1 6}}$.

Die modern- industrielle Entwicklung in Japan war eine Entwicklung ,,von oben“, und das sich modernisierende Meiji-Japan blieb auch nicht ,, autozentriert" im wirtschaftlichen Bereich wie das abgeschlossene Tokugawa-Japan autozentriert im wirtschaftlichen und im kulturellen Bereich gewesen war, sondern es griff nach außen - eine historische Tatsache, die auch Senghaas bei der Aufstellung seiner Hypothesen über die Entwicklung Japans bisher übersehen hat ${ }^{17}$.

Bereits 1876 zwang Japan Korea ,,Ungleiche Verträge“ auf, so wie sie Japan 1854/55 von den etablierten Weltmächten aufgezwungen worden waren, und damit begann praktisch schon die Vorbereitung zur kolonialen Unterwerfung Koreas (offiziell 1910). Einer der führenden Politiker Japans, Ito Hirobumi, erklärte bereits 1885, daß Japan ,,wegen seiner ständig wachsenden Bevölkerung" (!) Korea als ergänzende Reisquelle und zusätzliches Betätigungsfeld für die Söhne Japans brauche ${ }^{18}$. Gegen China 1894/95 und gegen Rußland 1904/05 führte Japan Kriege, die es gewann und die ihm territorialen Zuwachs, Geldmittel und internationales politisches Prestige einbrachten. Nach dem Sieg über China 1895 wurde Taiwan (Formosa) Japan zugesprochen, außerdem erhielt Japan von China eine „Entschädigung“ von 364 Millionen Yen, einer Summe, die ein Viertel des damaligen japanischen Nationaleinkommens ausmachte. 1897 konnte Japan den Goldstandard übernehmen und 1899 als erstes nicht-europäisches Land der Welt alle exterritorialen Rechte europäischer Mächte löschen $^{18}$. 1911 gewann Japan Tarifautonomie, und während des 1. Weltkrieges hatte es am asiatischen Handelsmarkt zu den Bedingungen einer Kolonialmacht teil.

Daß der 1885 konzipierte Plan zur Nutzung Koreas konsequent durchgeführt wurde, erweisen spätere Aufzeichnungen. Eine Kommission europäischer Wissenschaftler ${ }^{\mathbf{1 9}}$ stellte 1931/32 fest, daß aufgrund der Politik der japanischen Regierung in Korea Reis im Úbermaß angebaut und die Ernten überschüssig wurden, so daß die Reispreise steil abfielen; entsprechend stieg die Armut der koreanischen Reisbauern, sie konnten sich zum Überleben nur Hirse leisten. - Gemäß Eintrag in der Encyklopaedia Britannica betrug im Jahr 1939 der „,Export“ nach Japan $97 \%$ des Gesamtexportes Koreas und sein ,,Import“ aus Japan $89 \%$

\footnotetext{
16 Vgl. lenaga, Saburo: History of Japan. Tokyo 1953-1967.

17 Senghaas, Dieter: Japans dissoziativ-kapitalistische Entwicklung in: Weltwirtschaftsordnung und Entwicklungspolitik Plädoyer für Dissoziation 1977

18 Vgl. Wendt Die , unheimlichen" Japaner S. 110.

19 Sansom, G. B., und Kermode, D. W.: Economic Conditions in Japan to December 31st, 1932. With Annexes on Formosa and Korea. London 1933.
} 
des Gesamtvolumens. Korea ,,exportierte“ hauptsächlich Reis und Meeresprodukte und ,,importierte“ Fertigerzeugnisse ${ }^{20}$. Im Jahr 1939 waren Koreas Importe um 39 \% größer als seine Exporte, das Zahlungsdefizit wurde u. a. durch koreanische Gold,,verschiffungen“" ausgeglichen. Japan selbst wäre, so meint der Oxforder Japanologe Storry, in jener Zeit in Zahlungsschwierigkeiten gekommen, wenn es nicht einen großen Teil der benötigten Rohmaterialien aus Korea ,,importiert" hätte, dessen Industrie klein gehalten wurde ${ }^{\mathbf{2 1}}$. Japans Industrialisierung erfolgte also unter den gleichen Umständen wie die Europas, nämlich bei gleichzeitiger Expansion, und es ist in Anlehnung an den japanischen Fall umgekehrt zu überlegen, ob nicht auch in Europa die Industrialisierung weniger dank des Weberschen ,,mittelständischen Unternehmers“ möglich, sondern mehr eine „Entwicklung von oben“ war.

Wenn heute Analphabetismus, Bevölkerungszuwachs, „Traditionalismen“ verschiedener Art als entwicklungshemmend betrachtet werden, dann verwechselt man Ergebnisse der Industrialisierung mit deren Voraussetzungen, denn hinsichtlich dieser Faktoren sind die heutigen Entwicklungsländer in keiner anderen Situation, als es die heutigen Industrieländer zum entsprechenden Zeitpunkt waren. Die im Vergleich mit Europa größere Verbreitung der Schriftkundigkeit im 19. Jahrhundert in Japan kann kein wesentlicher Faktor bei der japanischen Industrialisierung gewesen sein, denn die Beherrschung der kultureigenen Schriftzeichen reichte zur Úbernahme europäischer Techniken ja nicht aus, man hätte europäische Sprachen verstehen müssen. Japanische Intellektuelle hatten zwar mit dem Studium des Holländischen während der Abschließungsepoche begonnen, aber es gab sicher noch mehr Inder, die während der Kolonialzeit das Englische in Wort und Schrift beherrschen lernten.

Entscheidende Unterschiede liegen in den jeweiligen inneren Gesamtsituationen der Länder und in den internationalen ,,Rahmenbedingungen“22, in denen sie stehen. In den europäischen Gesellschaften und in der japanischen waren im Gegensatz zu allen übrigen nicht-europäischen Gesellschaften am Beginn ihrer Industrialisierung ungebrochene autochthone Sozialstrukturen einschl. zwar armer, aber funktionsfähiger Landwirtschaften vorhanden, und parallel mit der europäischen Industrialisierung lief die koloniale Nutzung der nicht-europäischen Welt durch Europa, das für sich selbst die günstigsten Tarife erzwang und sich die gewünschten Märkte schuf. Das ungebrochene Japan konnte sich vor hundert Jahren als einzelnes Land im Kielwasser Europas expansiv in den Weltmarkt einschleusen ${ }^{23}$, angefochten genug wegen seines ,,Imitierens“ und seines ,,dumping“. Die heutigen Entwicklungsländer können sich nicht einschleusen; sie würden vielmehr zwangsläufig die internationalen Rahmenbedingungen verändern, wenn es ihnen gelänge, die gleichen Handelsbedingungen für sich zu schaffen, wie sie Europa und Japan am Anfang ihrer technisch-industriellen Entwicklung gehabt haben.

Die heutigen Entwicklungsländer können sich, bei der herrschenden internationalen Struktur, weder nach europäischem noch nach japanischem Vorbild entwickeln. Ihre wirtschaftliche Gesundung muß mit der Wiederherstellung ihrer nur noch fragmentarisch vorhandenen autochthonen Sozialstrukturen - also autozentriert - und buchstäblich auf grass roots' level,

20 Die Bedingungen des Warenaustauschs zwischen Kolonialherrscher und kolonial Beherrschtem sind zu verschieden von denen des Handels zwischen gleich starken Partnern, als daß die Begriffe „Export" und „,Import" hierfür geeignet wären.

21 Vgl. hierzu Storry, Richard: A History of Modern Japan. Middlesex 1960, 1965. Storry ist einer der wenigen Ostasien-Fachleute, die auch Korea behandeln.

$22 \mathrm{Vgl}$. in einem anderen Zusammenhang Bryde, Brun-Otto: Rezeption europäischen Rechts und autozentrierte Rechtsentwicklung in Afrika. Afrika Spektrum 1977.

23 Vgl. die Ausführungen über den japanischen Expansionismus jener Zeit des Harvard-Professors und langjährigen US-Botschafters in Tokyo Edwin O. Reischauer in: Japan Past and Present. Tokyo 1964, 1967. 
mit der Landwirtschaft, beginnen. Andernfalls bleiben sie immer in Gefahr, in strukturelle Despotie zu geraten und darin zwangsläufig zu stagnieren. Ob danach für sie eine Phase der Expansion, wie im Fall Japans, folgen kann und ob sie sich überhaupt nach dem Modell der europäisch industriellen Entwicklung wirtschaftlich regenerieren können, ist eine andere Frage. 


\section{Modern law and traditional societies}

BY FRANZ VON BENDA-BECKMANN

The analysis of the relationship between legal and socio-economic change requires a conceptual apparatus in which the concept of law is dissociated from the social processes to which its rules relate. The changes in the property and inheritance law of the Minangkabau of West Sumatra illustrate that important socio-economic changes may occur within the frame work of basically unchanged law. As is shown by a comparison between Minangkabau and Dutch law, the labels "modern" and "traditional/non modern" are highly misleading when applied to the unwritten law of non western societies and its development.

\section{The Role of the Judiciary in Modernizing Law in Africa By HeINRICH SCHOLleR}

The starts by describing the development of modern courts and modern law in Ethiopia which is characterized by a lack of colonial influence in this country. It shows the importance of mixed court jurisdiction in the early stage of modernizing law. This system had to resolve two mayor problems which involved the question of maintaining sovereignty: first, the jurisdiction over foreigners, second, the execution of foreign judgments in an African country. The problem of overcoming the concept of the so-called "duality of law" is also one of the main points of interest. The article stresses that the duality or plurality of law is not due to mere colonial policy, but rather to the process of modernizing law. The process of modernization started always out of a socially marginal position. This is also true for the revolutionary law although the role of courts and law during the Ethiopian revolution is only briefly mentioned.

According to this article the meaning of the term "modernizing law" is not restricted to the law making process itself but extended to the role of law as a part of social engineering. The independence of the law courts is considered an important element in the fulfilment of this function. The function of social engineering includes the task of education, assimilation and emancipation of men and women by modernized law. The task of political education can only be accomplished by law courts if they recognize their creative function and, for that purpose, claim a certain discretion in finding just and equitable solutions.

\section{“Autocentric" development in Ethno - Sociological perspective BY INGEBORG Y. WENDT}

Industrialization in Europe was pursued parallel with a growing philosophical inclination towards rationalism and materialism, i.e. economic development was in accordance with the general mental tendency. - There was wide-spread illiteracy even in England during the time of the "Industrial Revolution", and also rapid growth of population. These historical facts are ignored by theories on development, and wrongly literacy and family planning are considered as preconditions of economic growth in today's developing countries. - Japan, the only successful non-European country, was in seclusion when the rest of the non-European world was under colonial rule. Thus, it preserved its social system including a poor, but sustaining agriculture and its moral values, and retained its material substance. Japan was a well functioning, dynamic entity when it was forced to enter the international scene in $1853 / 54$. In contrast India, representing the former colonies and present developing coun- 
tries, was economically drained, its moral values were ignored by the colonial ruler, its social system including agriculture was destroyed. India entered international life, almost 100 years later than Japan, with a twofold, i.e. economic and psycho-sociological, deficit. - The Japanese epoch of seclusion was followed by a period of expansion. Japan won victories in wars with consequential gains of colonial territory and financial means as well as international political recognition and privileges. Although annoying the established world powers by "dumping" and "imitation", Japan as a single country could expand without changing the international economic frame. If India together with the other developing countries would create the same favourable international trade conditions for themselves now as Europa and Japan had at the start of their industrial development, the existing economic world system would necessarily change. - Before joining world trade under modern conditions, India would have to restore its autochthonic structure beginning with agriculture which was the given base at the start of industrialization in Europe and in Japan. Whether a period of expansion can follow like in case of Japan is doubtful.

\section{Concepts of development of local government administration in Nigeria By C. E. EMEZI}

The paper notes the importance attached to local government administration in Nigeria and briefly examines the traditional instruments of local administration in pre-colonial Nigeria. Thereafter, it attempts an overview of the concepts in the development of local government administration through four major epochs in Nigerian political history namely the colonial period, the period of decolonization, post-independence and post-civil war periods. One of the main thrust of local government reforms in Nigeria has been the swing of the pendulum from an attachment to traditional patterns of authority to a swing to elected representative councils.

The Federal Military Government, as a prelude to handing over power to civilians in Nigeria, gave the country a reformed uniform system of local government. There is the feeling that the reformed system may result in a conflict of roles between the traditional and modern instrumentalities of local government.

\section{The Israelis in Entebbe - Rescue or aggression? BY U. O. UMOZURIKE}

One hundred and five Israeli citizens in a plane hijacked to Entebbe were in great danger of losing their lives in the hands of Palestinians and their supporters. In view of President Amin's disdain for Israel, it was difficult to predict what he would have done even if Palestinian prisoners were released in exchange for the hostages. The right of a state to self-defence extends to the protection of citizens in great jeopardy in a foreign land. One prerequisite for the exercise of the right is that the imminent danger and the number of citizens must be proportional to the infringement of territorial sovereignty. Another prerequisite is that the foreign state must be either unwilling or incapable of protecting the foreigners. The principle of respect for fundamental human rights supports the right of humanitarian intervention to rescue national mortally endangered in a foreign land.

Israel was legally right to intervene an actions reasonably necessary for the exercise are justified but not actions in excess. The destruction of the radar system at Entebbe and the removal of expensive military equipments went beyond the requirements for the rescue and therefore ground liability for compensation. 